\title{
Single-Electron Model for Polaron on Dimerized Lattice
}

\author{
Astakhova T.Yu.*, Vinogradov G.A. ${ }^{\dagger}$ \\ N.M.Emanuel Institute of Biochemical Physics RAS, Moscow, Russia
}

\begin{abstract}
A new approach to modeling the electronic and dynamic properties of dimerized polyacetylene is proposed. Compared to the well-known and widely used approach, where dimerization is formed by the $\pi$-electrons of the valence band, in the proposed model the same effect is provided by an additional potential in the dynamic part of the Hamiltonian. In the usually used computational schemes, for a lattice of $N$ sites, $N \pi$-electrons have to be used to describe dimerization, so the total number of equations for quantum and classical dynamics is $\sim N^{2} / 2$. The integration step is dictated by the "fast" quantum subsystem and should be small. Both of these reasons make modeling difficult at large times and scales. In the proposed approach, in contrast to the $N$-electronic model, for describing a polaron on a dimerized lattice, the one-electron approximation is sufficient when the electron occupies the lowest level of the conduction band. Thus, the number of basis functions decreases to $\sim$ $3 N$, which allows to carry out calculations on large scales and times.
\end{abstract}

Key words: polaron, electron-phonon interaction, polyacetylene.

\section{INTRODUCTION}

Polyacetylene (PA) with the structural formula $(-\mathrm{CH}=\mathrm{CH}-)_{n}$ is an organic polymer with $\pi$-conjugated alternating single and double bonds. It plays an important role in both practical applications and especially in the theory of one-dimensional many-electron systems. Of greatest interest was the discovery of the transition of PA from a wide-gap semiconductor ( $\Delta E \approx 2.4 \mathrm{eV}$ ) to a conducting state with a specific conductivity comparable to the conductivity of metals $[1$, $\underline{2}, \underline{3}$ ] under doping (that is, in chemical contact with electron donors or acceptors).

Naturally, this discovery has generated great interest to the theory of many-electron systems and the reasons for the increase in conductivity by $\sim 10$ orders of magnitude upon doping. First of all, the reason for the bond alternation lengths was clarified. It was found that the effect is the result of competition between two forces acting on the carbon atoms of the PA polymer chain. The first arises due to the interaction of $\sigma$-electrons, and this force tends to equate bond lengths. Very often this interaction is taken into account in the harmonic approximation. The second force is provided by the conjugated $\pi$-electrons and tends to decrease bond lengths due to exchange interaction. It is a compromise between these two forces that determines the equilibrium with alternating bonds. This effect is sometimes referred to as the Peierls' alternation [4].

There are two main approaches to description of the PA lattice dimerization. In the first, dimerization is introduced through the so-called symmetry breaking term, first proposed by S. Brazovsky and N. Kirova $[\underline{5}, \underline{6}]$. The second method uses a model that includes all $\pi$-electrons. If the PA chain is neutral ( $N$ electrons at $N$ lattice sites), then the electrons occupying the valence band provide the lattice dimerization.

Currently, it is believed that the conductivity of PA is implemented by polarons. Polarons are formed as a result of doping, when the dopant, an electron acceptor or donor, takes or gives

\footnotetext{
*astakhova@deom.chph.ras.ru

†gvin@deom.chph.ras.ru
} 
an extra electron to the PA chain. The joint evolution of the dynamic and quantum subsystems in the presence of an extra charge (electron or hole) results in the formation of a polaron and this corresponds to a minimum of the total energy of the system.

Following the polaron paradigm in the explanation of the PA conductivity, it is necessary to understand the polaron dynamics and mechanisms of the polaron interaction with the external field. Research in this area began about 40 years ago with the study of charged solitons in PA $[\underline{7}, \underline{8}, \underline{9}, \underline{10}]$. Later attempts were made to reveal the effect of the applied electric field on the charged soliton [11].

This issue was considered in more detail in $[12,13,14,15]$. Later, many works were devoted to the dynamics of polaron in an electric field. One of the most intriguing features was a step-like change in the polaron velocity from the subsonic values to those exceeding the speed of sound by a factor of $\approx 3$ within a very small range of the electric field strength $[16,17,18]$.

To describe a polaron, a joint consideration of the classical lattice dynamics and quantum charge dynamics is necessary. Classical dynamics are Newtonian equations. Quantum dynamics is most often formulated on the basis of the $\mathrm{Su}$, Schrieffer, and Heeger ( $\mathrm{SSH}$ ) approximation $[\underline{8}, 9]$, which is based on the tight-binding (TB) approximation when interaction only between the nearest neighbors is taken into account. The combination of classical and quantum methods in one problem raised some questions. These issues were resolved when the lattice was quantized [19].

The unit cell in PA is dimerized (consists of two sites) and, therefore, there are two branches in the vibrational spectrum, acoustic and optical. It is the transition of the generated oscillations from the acoustic to the optical branch that phenomenologically explains the step-like dependence of the polaron velocity on applied field strengths.

From a computational point of view, the many-electron problem is time consuming as it is necessary to integrate the system of equations for $\sim N^{2} / 2$ variables with a small "quantum", time integration step. Usually the system of several hundred particles and cyclic boundary conditions are used. In this paper, we propose a single-electron model of PA, which fully preserves the main features of many-electron model, but the number of variables decreases to $\sim 3 N$.

\section{MODELS DESCRIPTION}

\section{Many-electron model}

In this section we briefly recall the many-electron (ME) model which is usually applied for modeling polarons in PA and other conjugated polymers.

Usually the Hamiltonian of a polaron model has two terms

$$
H=H_{\mathrm{el}}+H_{\mathrm{lat}},
$$

where the electronic quantum term $H_{\mathrm{el}}$ acts on the wave functions of the electrons and is also responsible for the electron-phonon interaction. In the case of PA, there are $N \pi$-electrons fully occupying $N / 2$ levels of the valence band and extra $(N+1)$ st electron at the lowest level of the conducting band. The classical term $H_{\text {lat }}$ in (1) describes the interaction between the lattice units.

When modeling polarons in PA, $H_{\mathrm{el}}$ is usually taken in the $\mathrm{SSH}$ approximation:

$$
H_{\mathrm{el}}=-\sum_{j}\left[t_{0}-\chi\left(y_{j}-r_{0}\right)\right]\left[\mathrm{e}^{i \gamma A(t)} c_{j+1}^{+} c_{j}+H . c .\right] .
$$

Here $y_{j} \equiv x_{j+1}-x_{j}$, where $x_{j}$ is the absolute coordinate of $j$-th $\mathrm{CH}$ group along the lattice, and all $y_{j}=r_{0}$ for the optimized undimerized lattice, where $r_{0}$ is the lattice constant; $c_{j}^{+} / c_{j}$ is the 
creation/annihilation operator of an electron at the site $j, t_{0}$ is the hopping integral at equilibrium, and $\chi\left(y_{j}-r_{0}\right)$ is its modification due to the lattice displacement, $A(t)$ is the time-dependent vector potential and the electric field is given by $E=-\frac{1}{c} \frac{d A}{d t}$. The parameter $\gamma$ is defined as $\gamma=e r_{0} / \hbar c$, where $e$ is the absolute value of the electronic charge, $c$ is the light velocity, and $\hbar$ is the reduced Planck constant. In the case of constant electric field $A(t)=-c E t$ and exponential factor in (2) transforms as $\exp [i \gamma A(t)] \rightarrow \exp \left(-\frac{i e E r_{0}}{\hbar} t\right)$.

Matrix elements of (2) for cyclic boundary conditions are:

$$
\begin{aligned}
H_{j, j+1} & =H_{j+1, j}=-\left[t_{0}-\chi\left(y_{j}-r_{0}\right)\right] ; \\
H_{1, N} & =H_{N, 1}=-\left[t_{0}-\chi\left(y_{N}-r_{0}\right)\right] ; \quad H_{j, j}=0 .
\end{aligned}
$$

The lattice Hamiltonian in ME model of PA is

$$
H_{\mathrm{lat}}^{\mathrm{ME}}=\frac{k_{0}}{2} \sum_{j}\left(y_{j}-r_{0}\right)^{2}+\frac{M}{2} \sum_{j} \dot{x}_{j}^{2},
$$

where $M$ is the mass of the $\mathrm{CH}$ unit, $k_{0}$ is the spring constant for the $\sigma$ energy when expanded to the second order about the equilibrium undimerized system.

Thus, in the ME model, the classical lattice Hamiltonian (4) describes an undimerized lattice with an equilibrium distance between sites $r_{0}$. The quantum Hamiltonian (2) refers to the interaction of $\pi$-electrons with the lattice, leading to lattice dimerization.

Cyclic boundary conditions are usually used. For the charged lattice of $N$ sites, the number of electrons is $N+1$, i.e. $N / 2$ levels of the valence band are double occupied, and the extra electron occupies the lowest level of the conduction band.

The generally used parameters for PA model are $k_{0}=21 \mathrm{eV} / \AA^{2}, t_{0}=2.5 \mathrm{eV}, \chi=4.1 \mathrm{eV} / \AA$, $r_{0}=1.22 \AA, M=13 \mathrm{amu}[\underline{20}]$. The parameters are fitted to correctly describe the geometry and band structure of the perfect dimerized lattice.

Usually, when modeling the polaron evolution in the electric field, a static polaron is formed as the initial conditions. And then the electric field is switched on instantly or otherwise. The static polaron solution is obtained by solving the self-consistent system of equations for $y_{j}$ :

$$
y_{j}=r_{0}-\frac{2 \chi}{K} \rho_{j, j+1}+\frac{2 \chi}{K N} \sum_{j^{\prime}}^{\mathrm{occ}} \rho_{j^{\prime}, j^{\prime}+1}
$$

where $\rho_{j, j+1}=\sum_{v} \psi_{v, j} \psi_{v, j+1}^{*}$ is the matrix element of the density matrix $\rho$ and $\Psi_{v}$ is the eigenfunction diagonalizing the electronic Hamiltonian $H_{\mathrm{el}}$ with $A=0$, when the eigenfunctions can be taken as real functions. The summation over $v$ is restricted to the occupied states. Equations (5) are obtained by minimizing the energy described by the Hamiltonian (2) and (4) with the additional condition $\sum y_{j}=N r_{0}$ which is necessary to satisfy the cyclic boundary conditions. Equations (5) can be used since here the lattice coordinates $y_{j}$ are explicitly expressed in terms of electronic wave functions.

The polaron dynamics in an external electric field is described by joint solving the equations following from (2) and (4) (for more details see e.g. [12]). In the ME model, it is necessary to integrate $\sim \bar{N}^{2} / 2$ equations for electronic and dynamic variables. In addition, the time integration step should be small due to the "fast" quantum subsystem. Therefore, due to computational difficulties, a relatively small number of particles and cyclic boundary conditions are usually used. This does not allow a detailed analysis of the polaron dynamics at large times and scales. For this reason, an attempt is made to formulate a simpler single-electron model 
preserving the basic features of the ME problem.

\section{Single-electron model}

When constructing a single-electron model (SE) of PA, it is necessary to solve the main problem - to provide the dimerization of the lattice while maintaining the basic dynamic features of the ME model. In the ME model, the interaction of $\pi$-electrons is described in the TB approximation taking into account the lattice deformation. Effectively, this interaction causes compression of the lattice. The competition between the harmonic lattice interaction and the interaction of $\pi$-electrons results into the lattice dimerization. The ME model has a clear physical background and well describes the dynamic and electronic properties of PA.

The dimerization in the proposed SE model is described by an additional artificial classical potential simulating the lattice compression due to the interaction of $\pi$-electrons. This is possible due to the following non-obvious property of the ME model. It turned out that in the ME model, the electrons of the valence band do not make any contribution to the formation of the polaron when an extra electron is added. An analysis of the wave functions density distribution over the lattice sites shows that for both the standing and moving polarons, at any lattice site the average charge density resulted from $N$ electrons of the valence band is 1 . And the charge localization and lattice deformation are provided exclusively by an extra electron occupying the lowest level of the conduction band. This fact allows to construct a model in which the dimerization is provided by the classical potential with appropriately fitted parameters.

The lattice Hamiltonian in the SE model is

$$
H_{\mathrm{lat}}^{\mathrm{SE}}=\frac{k_{0}}{2} \sum_{j=1}^{N}\left(y_{j}-r_{0}\right)^{2}+\frac{k_{1}}{2} \sum_{j=1, N / 2}^{\text {single }} y_{2 j-1}^{2}+\frac{k_{2}}{2} \sum_{j=1, N / 2}^{\text {double }} y_{2 j}^{2}+\frac{M}{2} \sum_{j} \dot{x}_{j}^{2},
$$

where the notations are the same as above.

The first term in right-hand side of Eq. (6) is the same as in the ME model (see Eq. (4)). It describes the interaction of $\sigma$ electrons and is responsible for the lattice tendency to the undimerized state with $y=r_{0}$. The second and the third terms describe the compression of the lattice due to the interaction of $\pi$-electrons and have a minimum at $y=0$. The lattice with this interaction potential, has a dimerized equilibrium state with alternating single (long) and double (short) bonds. For definiteness, we assume that odd bonds are single, and even bonds are double, i.e. $k_{2}>k_{1}$. Then the second sum in (6) refers to the additional potential for single odd bonds (the index is "single" when summing), and the third sum refers to the potential for double even bonds (index "double").

The potential described by the Hamiltonian Eq. (6) has an analytical solution for the equilibrium bond lengths with $r_{1}=r_{0}+\delta$ for single bonds, and $r_{2}=r_{0}-\delta$ for double bonds. Here, $\delta$ is the alternation parameter

$$
\delta=\frac{k_{2}-k_{1}}{2 k_{0}+k_{1}+k_{2}} r_{0}
$$

Note that the Eqs. (7) are valid for cyclic boundary conditions, where the condition $\sum_{j} y_{j}=$ $N r_{0}$ is satisfied. Note that the lattice interaction between two nearest sites in the SE model is effectively harmonic with the potential $\sum_{j=1}^{N} \frac{k_{j}}{2}\left(y_{j}-r_{j}^{e q}\right)^{2}$ with renormalized alternating parameters, namely, $k_{j}=k_{0}+k_{1}$ and $r_{j}^{e q}=r_{1}$ for odd $j$ (single bonds) and $k_{j}=k_{0}+k_{2}$, and $r_{j}^{e q}=r_{2}$ for even $j$ (double bonds). Note that with the same scaling of $k_{0}, k_{1}$ and $k_{2}, r_{1}$ and $r_{2}$ do not change.

The parameters of the SE model are summarized in Table 1 . The values of $M$ and $r_{0}$ 
Table 1. SE model parameters

\begin{tabular}{|c|c|}
\hline parameter & value \\
\hline$k_{0}, \mathrm{eV} / \AA^{2}$ & 7.5 \\
\hline$k_{1}=k_{0}, \mathrm{eV} / \AA^{2}$ & 6.5 \\
\hline$k_{2}=k_{0}, \mathrm{eV} / \AA^{2}$ & 8.5 \\
\hline$t_{0}, \mathrm{eV}$ & 2.5 \\
\hline$\chi, \mathrm{eV} / \AA^{2}$ & 6.0 \\
\hline$r_{0}, \AA$ & 1.22 \\
\hline$M, \mathrm{amu}$ & 13 \\
\hline
\end{tabular}

are obviously the same as in the ME model. The spring constants are selected from the best coincidence of the vibrational spectra of both models providing the correct bond lengths of the perfect dimerized lattice. Note that the ME model for uncharged lattice include only pairwise interaction. So only the coincidence of the general view of the vibrational spectra and the close values of frequencies can be achieved. The $t_{0}$ value is taken the same as in the ME model. The $\chi$ value is chosen so that the geometry of the standing polaron (amplitude and width) to be similar in both models.

The geometry of standing polaron in the SE model is derived by numerically solving an equation similar to Equation (5)

$$
\begin{aligned}
y_{2 j-1} & =\tilde{k_{2}} r_{0}-\frac{2 \chi}{k_{0}+k_{1}} \rho_{2 j-1,2 j}+\frac{2 \chi}{N} \tilde{k_{2}} \tilde{\rho} \\
y_{2 j} & =\tilde{k_{1}} r_{0}-\frac{2 \chi}{k_{0}+k_{2}} \rho_{2 j, 2 j+1}+\frac{2 \chi}{N} \tilde{k_{1}} \tilde{\rho}
\end{aligned}
$$

where

$$
\begin{aligned}
\tilde{k_{1}} & =\frac{2\left(k_{0}+k_{1}\right)}{2 k_{0}+k_{1}+k_{2}} ; \quad \tilde{k_{2}}=\frac{2\left(k_{0}+k_{2}\right)}{2 k_{0}+k_{1}+k_{2}} \\
\tilde{\rho} & =\frac{1}{k_{0}+k_{1}} \sum_{j^{\prime}=1}^{N / 2} \psi_{2 j^{\prime}-1} \psi_{2 j^{\prime}}+\frac{1}{k_{0}+k_{2}} \sum_{j^{\prime}=1}^{N / 2} \psi_{2 j^{\prime}} \Psi_{2 j^{\prime}+1}
\end{aligned}
$$

and $\{\Psi\}$ is the wave function of the lowest level of the conduction band.

The polaron evolution is derived by joint numerical solution of dynamical equations for the lattice displacements

$$
\begin{aligned}
M \ddot{x}_{2 j-1}= & k_{0}\left(2 x_{2 j-1}-x_{2 j}-x_{2 j-2}\right)+k_{1}\left(x_{2 j}-x_{2 j-1}\right)-k_{2}\left(x_{2 j-1}-x_{2 j-2}\right) \\
& +\chi\left\{F\left[\psi_{2 j-1}^{*} \psi_{2 j}-\psi_{2 j-2}^{*} \psi_{2 j-1}\right]+\text { c.c. }\right\} \\
M \ddot{x}_{2 j}= & k_{0}\left(2 x_{2 j}-x_{2 j+1}-x_{2 j-1}\right)+k_{2}\left(x_{2 j+1}-x_{2 j}\right)-k_{1}\left(x_{2 j}-x_{2 j-1}\right) \\
& +\chi\left\{F\left[\psi_{2 j}^{*} \psi_{2 j+1}-\psi_{2 j-1}^{*} \psi_{2 j}\right]+\text { c.c. }\right\}
\end{aligned}
$$

and the time-dependent Schrödinger equation for the wave function

$$
i \hbar \frac{\partial \psi_{j}}{\partial t}=-\left[t_{0}-\chi\left(x_{j+1}-x_{j}-r_{0}\right)\right] F \psi_{j+1}-\left[t_{0}-\chi\left(x_{j}-x_{j-1}-r_{0}\right)\right] F^{*} \psi_{j-1},
$$


where $F=\exp \left(-\frac{i e E r_{0}}{\hbar} t\right)$. Equations (9) and (10) are integrated by the Runge-Kutta method with a time step of $10^{-2}$ fs.

\section{MODELS COMPARISON}

In this section we liken together the main results for two models. First of all, we compare the vibrational spectra of dimerized lattices in both models, since the vibrational spectrum is an important characteristic, and it is also believed that it determines the polaron evolution in the electric field.

Note that we do not try to choose the values of the SE model parameters so as to fully correspond to the ME model, which is not possible. Therefore, one cannot expect a complete coincidence of the results for both models. It is important that the simplified SE model qualitatively reproduces the basic features of the original ME model.

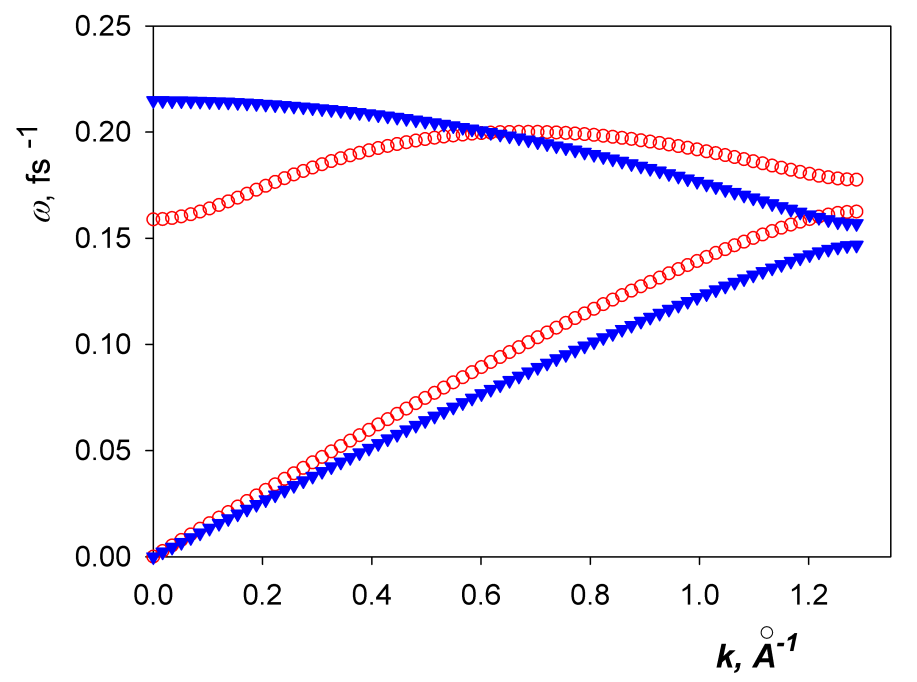

Fig. 1. Vibrational spectra of uncharged dimerized lattice for the ME (open red circles) and SE (filled blue triangles) models. In both cases, the lower curve is the acoustic branch and the upper curve is the optical branch.

The vibrational spectra of uncharged dimerized lattice of $N=300$ sites for both models are shown in Figure 1. The spectrum is derived by the diagonalization of the Hessian which is the matrix of second derivatives of the total energy $U$ of the perfect dimerized uncharged lattice. For the ME model, the matrix elements are calculated numerically: $\operatorname{Hess}_{i, j}=\partial^{2} U / \partial x_{i} \partial x_{j}=$ $-\partial f_{i} / \partial x_{j}$, where $f_{i}$ is the $i$-th component of the force. For the SE model, the matrix elements have simple expressions:

$$
\text { Hess }=\left(\begin{array}{ccccc}
\cdots & \ldots & \ldots & \ldots & \ldots \\
\cdots & 2 k_{0}+k_{1}+k_{2} & -\left(k_{0}+k_{1}\right) & 0 & \ldots \\
\ldots & -\left(k_{0}+k_{1}\right) & 2 k_{0}+k_{1}+k_{2} & -\left(k_{0}+k_{2}\right) & \ldots \\
\ldots & 0 & -\left(k_{0}+k_{2}\right) & 2 k_{0}+k_{1}+k_{2} & \ldots \\
\ldots & \ldots & \ldots & \ldots & \ldots
\end{array}\right) \leftarrow 2 i-1
$$

A characteristic feature of the spectrum is the weak dependence of the optical frequency on the wave vector $k$. For the ME model, the optical branch has a wide and smooth maximum at $k \approx 0.7 \AA^{-1}$. The nonmonotonic dependence of the optical frequency on $k$ is related to 
the long-range nature of the electron-phonon interaction between the lattice and $N$ electrons occupying $N / 2$ levels of the valence band.

The SE model for an uncharged lattice includes only pairwise interaction of neighboring sites. In this case, the maximum of the optical vibrations branch is in the center of the Brillouin zone $(k=0)$. Despite these insignificant differences, the general patterns of the spectra are similar for both models.
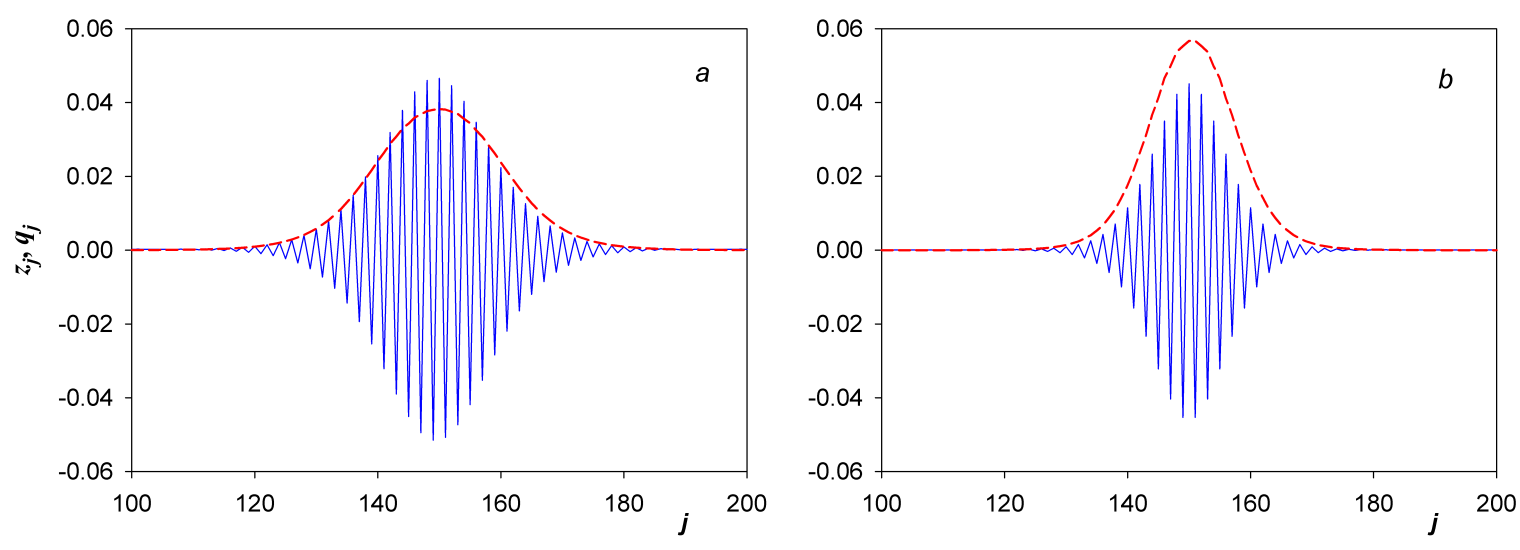

Fig. 2. Standing polaron for the ME (a) and SE (b) models.

Figure 2 shows standing polarons derived by numerical solutions of Equations (5) and (8) for ME and SE models, respectively. One can see that they are very similar. Here, the lattice geometry is expressed in terms of bond deviation defined as $z_{j} \equiv x_{j+1}-x_{j}-r_{0}$, where $r_{0}$ is the $j$-th bond length in the dimerized lattice for either the single bond $\left(r_{0}^{\mathrm{s}} \approx 1.14 \AA\right)$ or the double one $\left(r_{0}^{\mathrm{d}} \approx 1.3 \AA\right), z_{j}$ is positive for double bonds, and negative for single bonds, i.e. short double bonds get longer in the polaron domain, and long single bonds are shortened.

For the ME model, $q_{j}$ is defined as the averaged additional charge $q_{j} \equiv\left[\tilde{\rho}_{j+1}+\tilde{\rho}_{j-1}+2 \tilde{\rho}_{j}\right] / 4$, where $\tilde{\rho}_{j}=\sum_{v}\left|\psi_{v, j}\right|^{2}-1$ with summation over the occupied levels $(\nu \in[0, N / 2+1])$. In the case of the SE model, $q_{j}$ is the average charge per bond between sites $j$ and $j+1$, i.e. $q_{j}=0.5\left(\left|\psi_{j}\right|^{2}+\left|\psi_{j+1}\right|^{2}\right)$.

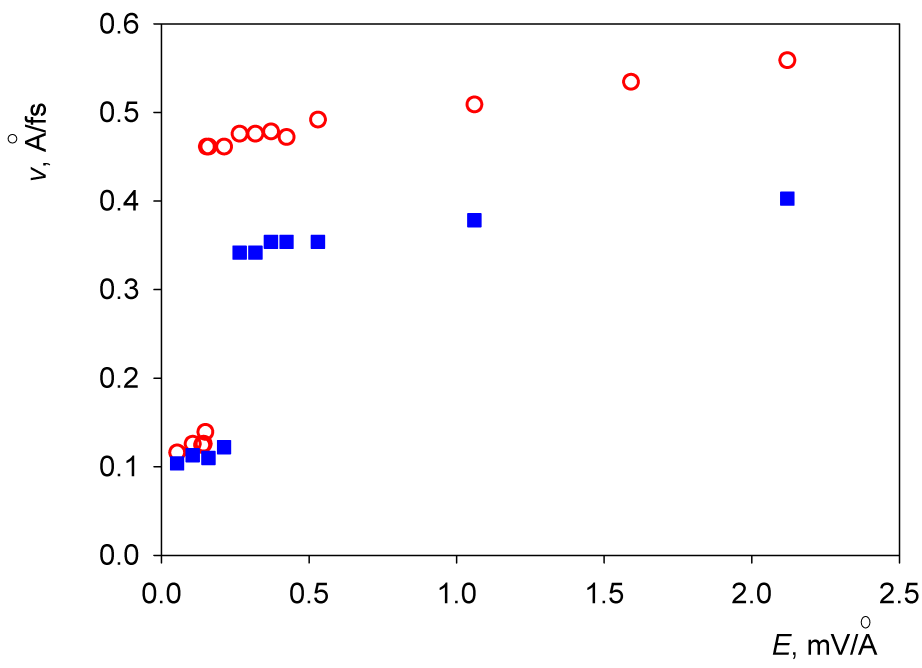

Fig. 3. Dependence of polaron velocity on electric field strength: the ME model (open red cicles) and the SE model (filled blue squares). 
Since polarons are of primary interest as charge carriers, a huge amount of work has been devoted to modeling the evolution of polarons in an electric field. A characteristic feature of the polaron behavior in the electric field in the ME model is a stepwise dependence of the stationary polaron velocity on the field strength (see curve of circles in Fig. 3). This effect is phenomenologically explained by the presence of two vibrational branches, acoustic and optical, and the transition between them.

For the ME model, a direct relationship between the behavior of a polaron in an electric field and the vibrational spectrum of an uncharged lattice was demonstrated in molecular dynamical simulations [21]. It was shown that the range of allowed velocities of subsonic polarons corresponds to the range of phase velocities of the acoustic vibrational branch, while the speed of supersonic polarons lies in the range of phase velocities of the optical vibrational branch. Moreover, a moving supersonic polaron generates monochromatic oscillations representing a plane wave. These vibrations are one of the eigenmodes of the optical vibrational branch, and the phase velocity of this mode is equal to the phase velocity of the polaron. However, the reason for the step in the dependence of polaron on the electric field strength (from $v_{\text {snd }}$ to about $4 v_{\text {snd }}$ ) remains unclear, since there is no gap between the maximum phase velocity of the acoustic branch $\left(v_{\text {snd }}\right)$ and the minimum phase velocity of the optical branch.

Since the parameters of the SE model are selected so that the vibrational spectra in both models are similar, we can expect a similar dependence of the polaron velocity on the electric field strength, as Figure $\underline{3}$ demonstrates.
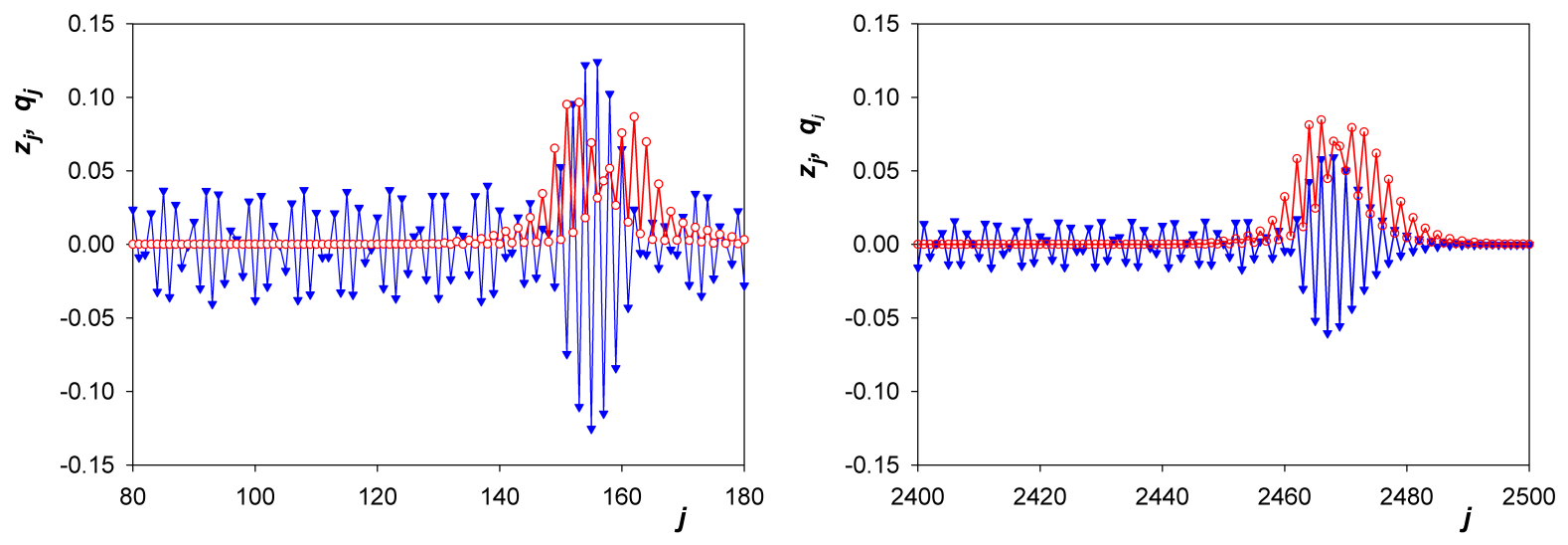

Fig. 4. Snapshots of the polaron evolution for the ME (left panel) and SE (right panel) models. Here, bond deviations $z_{j}$ are shown by filled blue triangles and and charge distribution $\left|\psi_{j}\right|^{2}$ is shown by open red circles.

For illustration, we compare the results of molecular dynamics modeling for both models. Figure 4 shows lattice fragments of 100 sits with a polaron moving in an electric field. The polaron velocity is the same and equal to $3.9 v_{\text {snd. }}$. Left panel of Figure $\underline{4}$ presents the ME model. Here the lattice length is 300 sites, the polaron is initially centered at site $150, z_{j}$ is the bond deviation as above, and $\left|\psi_{j}\right|^{2}$ is the charge distribution of the extra electron. The snapshot is at $t=2000 \mathrm{fs}$. At this moment, the polaron moved around the lattice three times and generated vibrations are superimposed on its profile. Right panel of Figure $\underline{4}$ presents the SE model. Here the lattice length is 3000 sites, the polaron is also initially centered at site $150, z$ is the bond deviation as well, and $|\psi|^{2}$ is the charge distribution of the single electron. The snapshot is presented at $t=6000 \mathrm{fs}$. By this time, the polaron has not yet reached the end of the chain. A comparison of the figures shows a similar evolutionary pattern for both models. Moreover, due to the large lattice length in the SE model, it is possible to analyze the evolution of the polaron profile over a long time. 
In addition the simplicity of the SE model gives hope for the development of an analytical model similar to that for polaron on harmonic lattice, and then to analyze the polaron evolution in an electric field.

\section{CONCLUSIONS}

A new approach is proposed for describing the polaron evolution on the dimerized PA lattice. To date, the many-electron SSH model was used. The physically clear and simple approximation for the electron Hamiltonian, combined with a simple description of the lattice dynamics, made this model very attractive for research. Within the framework of this model, an astonishing result was obtained that the polaron velocity in an external electric field increases approximately 3 times, to speeds exceeding the speed of sound, with a very small change in the field strength. Although it has been suggested that the jump in speed is associated with the transition from the acoustic to the optical branch of vibrational excitations, there is still no clear understanding of the nature of this phenomenon.

Unfortunately, the SSH model is time and memory consuming in numerical simulations, as it includes $N(N / 2+1)$ quantum variables for electron wave functions and $2 N$ classical variables for the lattice coordinates and velocities. That is why the size of modeled lattice is usually about several hundreds sites and cyclic boundary conditions are used. However, there is no certainty that with a limited simulation time, the system achieves a stationary state.

In the proposed SE model, dimerization is taken into account through an additional term in the classical lattice potential. The parameters of the model are selected in such a way that it preserves the main features of the ME problem. It is hoped that the use of the proposed SE model will elucidate the problem of the step-like dependence of polaron velocity on electric field strength. Since the simplicity of the model gives us hope to get an analytical solution for a moving polaron.

\section{REFERENCES}

1. Heeger A.J. Nobel Lecture: Semiconducting and metallic polymers: The fourth generation of polymeric materials. Rev. Mod. Phys. 2001. V. 73. № 3. P. 681-700. doi: 10.1103/RevModPhys.73.681.

2. MacDiarmid A.G. Nobel Lecture: "Synthetic metals": A novel role for organic polymers. Rev. Mod. Phys. 2001. V. 73. № 3. P. 701-712. doi: 10.1103/RevModPhys.73.701 .

3. Shirakawa H. Nobel Lecture: The discovery of polyacetylene film - the dawning of an era of conducting polymers. Rev. Mod. Phys. 2001. V. 73. № 3. P. 713-710. doi: 10.1103/RevModPhys.73.713.

4. Peierls R.E. Quantum Theory of Solids. Oxford Scholarship Online: September 2007. Chapter 5 Cohesive Forces in Metals. doi: 10.1093/acprof:oso/9780198507819.003.0005.

5. Brazovskii S.A. Self-localized excitations in the Peierls-Fröhlich state.Sov. Phys. JETP. 1980. V. 51. № 2 P. 342-353.

6. Brazovskii S.A., Kirova N.N. Exitons, polarons, and bipolarons in conducting polymers. JETP Letters. 1981. V. 33. № 1. P. 4-8.

7. Su W.P., Schrieffer J.R., Hegger A.J. Solitons in polyacetylene. Phys. Rev. Lett. 1979. V. 42. № 25. P. 1698-1701. doi: 10.1103/PhysRevLett.42.1698.

8. Su W.P., Schrieffer J.R. Soliton dynamics in polyacetylene. Proc. Natl. Acad. Sci. U.S.A. 1980. V. 77. № 10. P. 5626-5629. doi: 10.1073/pnas.77.10.5626.

9. Su W.P., Schrieffer J.R., Heeger A.J. Soliton excitations in polyacetylene. Phys. Rev. B. 1980. V. 22. № 4. P. 2099-2105. doi: 10.1103/PhysRevB.22.2099.

10. Takayama H., Lin-Liu Y.R., Maki K. Continuum model for solitons in polyacetylene. Phys. Rev. B. 1980. V. 21. № 6. P. 2388-2393. doi: 10.1103/PhysRevB.21.2388. 
11. Wilson E.G. The dynamics and stability of the solitary wave acoustic polaron. Synthetic Metals. 1989. V. 28. № 3. P. D551-D556. doi: 10.1016/0379-6779(89)90743-1.

12. Ono Y., Terai A. Motion of charged soliton in polyacetylene due to electric field. J. Phys. Soc. Jpn. 1990. V. 59. № 8. P. 2893-2904. doi: 10.1143/JPSJ.59.2893.

13. Arikabe Y., Kuwabara M., Ono Y. Dynamics of an acoustic polaron in one-dimensional electron-lattice system. J. Phys. Soc. Jpn. 1996. V. 65. № 5. P. 1317-1324. doi:10.1143/JPSJ.65.1317.

14. Johansson A., Stafström S. Polaron dynamics in a system of coupled conjugated polymer chains. Phys. Rev. Lett. 2001. V. 86. № 16. P. 3602-3605. doi: 10.1103/PhysRevLett.86.3602.

15. Korshunova A.N., Lakhno V.D. Tech. Phys. 2018. V. 63. P. 1270. doi: 10.1134/S1063784218090086.

16. Johansson A.A., Stafström S. Nonadiabatic simulations of polaron dynamics Phys. Rev. B. 2004. V. 69. P. 235205. doi: 10.1103/PhysRevB.69.235205.

17. Ribeiro L.A., da Cunha W.F., de Oliveria Neto P.H., Gargano R., e Silva G.M. Effects of temperature and electric field induced phase transitions on the dynamics of polarons and bipolarons. New J. Chem. 2013. V. 37. P. 2829-2836. doi: 10.1039/c3nj00602f.

18. Astakhova T.Yu., Vinogradov G.A. Polaron in Electric Field and Vibrational Spectrum of Polyacetylene. Mathematical Biology and Bioinformatics. 2019. T. 14. No 1. C. 150-159. doi: $10.17537 / 2019.14 .150$.

19. Likhachev V.N., Vinogradov G.A. Quantum problem of polaron localization and justification of the Su-Schrieffer-Heeger approximation. Theor. Math. Physics. 2019. V. 199. № 2. P. 719-725. doi: 10.1134/S0040577919050088.

20. Ono Y., Terai A. Motion of charged soliton in polyacetylene due to electric field. Synthetic Met. 1991. V. 43. № 3. P. 3689-3692. doi: 10.1016/0379-6779(91)91661-S.

21. Astakhova T., Vinogradov G. New aspects of polaron dynamics in electric field. Eur. Phys. J. B. 2019. V.92: 247. doi: 10.1140/epjb/e2019-100339-y.

Accepted 15 November 2019.

Revised 15 December 2019.

Published 17 December 2019. 